\section{Periodontitis agresiva. Reporte de caso}

López-Pinedo ML, Hidalgo-Medina E. Periodontitis agresiva. Reporte de caso. Rev Estomatol Herediana. 2010; 20(4):212-215.

\section{RESUMEN}

La periodontitis agresiva es una infección con baja prevalencia, caracterizada clínicamente por pérdidas rápidas de inserción y radiográficamente por reabsorciones óseas severas, que se presentan tempranamente alrededor de la infancia en niños y adultos jóvenes sanos. El presente reporte de caso describe a un paciente de sexo masculino de trece años de edad, el cual acude a la consulta odontológica por presentar movilidad y separación de piezas dentarias especialmente en el sector antero superior e inferior, sangrado gingival al cepillado y dolor a la masticación. Clínicamente se observó aumento de volumen y cambio de coloración gingival, presencia de bolsas periodontales profundas, baja presencia de placa y/o cálculo. Radiográficamente se confirmó la presencia de reabsorciones óseas severas localizadas a nivel de los primeros molares e incisivos. El tratamiento no quirúrgico consistió en el raspado y alisado radicular dado con terapia antibiótica y antiséptica adjunta. A las 6 semanas post tratamiento se observó una mejoría en los niveles de inserción clínica, con la consecuente disminución de las bolsas periodontales, e inflamación gingival así como ausencia de sangrado; el paciente pudo lograr un óptimo control de su higiene oral. Debido a que esta enfermedad periodontal es altamente destructiva de los tejidos periodontales que soportan los dientes, es de suma importancia su detección temprana por parte de los profesionales de la salud oral.

Palabras clave: PERIODONTITIS / PERIODONTITIS AGRESIVA / Aggregatibacter actinomycetemcomitans.

\section{Aggressive periodontitis. A case report}

ABSTRACT

The aggressive periodontitis is an infection, with low prevalence, characterized clinically by rapid attachment loss and radiographically by severe bone resorption, which can occur as early as in children and young adults. This case report describes a 13 year old male patient, who went to the dental office for mobility and separation of teeth especially in the upper and lower anterior teeth, gingival bleeding when brushing and pain on mastication. The clinical exam revealed swelling and gingival discoloration, presence of deep periodontal pockets, and low presence of plaque and/or dental calculus. Radiographically the presence of localized severe bone resorption at the level of first molars and incisors was found. The treatment consisted of scaling and root planning, antibiotic and antiseptic therapy. At 6 weeks after treatment, it was observed an improvement in clinical attachment levels, with consequent reduction in periodontal pockets and gingival inflammation and absence of bleeding. The patient was able to achieve an optimal control of oral hygiene. Because periodontal disease is highly destructive of the periodontal tissues that support the teeth, is crucial early detection by oral health professionals. Key words: PERIODONTITIS / AGGRESSIVE PERIODONTITIS / Aggregatibacter actinomycetemcomitans.

\section{Martha L. López Pinedo' Elizabeth Hidalgo Medina²}

'Docente del Departamento Académico de Clinica Estomatológica.

${ }^{2}$ Estudiante de Pregrado.

Facultad de Estomatología. Universidad Peruana

Cayetano Heredia.

\section{Correspondencia}

Martha L. López Pinedo

Av. Honorio Delgado 430 - Lima 31, Perú

Teléfono (511)381-1950 - Anexo 253

e-mail: martha.lopez@upch.pe

marlop99@gmail.com

Recibido : 20 de mayo de 2010

Aceptado : 15 de setiembre de 2010

\section{Introducción}

La periodontitis agresiva afecta a las personas que en muchos casos parecen estar sanos, excepto por la presentación de la periodontitis; antes de 1999 se denominaba Periodontitis de aparición temprana y su diagnóstico se basaba en la edad del paciente y en el tiempo de presentación de la enfermedad (1).

En 1999, en el Taller Internacional para una Clasificación de las Condiciones y Enfermedades Periodontales, organizado por la Academia Americana de Periodoncia, se cambió el nombre de periodontitis de aparición temprana por el de Periodontitis Agresiva (PA), determinándose que su diagnóstico debe darse en relación a los hallazgos clínicos, radiográficos y de laboratorio. Estos hallazgos la hacen suficientemente diferente de la periodontitis crónica (2).

La PA tiende a tener una agregación familiar y una rápida progresión de la enfermedad y suele presentarse antes de los 30 años (3), y se divide en localizada y generalizada.

El término de periodontitis agresiva localizada reemplaza al de periodontitis juvenil localizada y el de periodontitis agresiva generalizada al de periodontitis juvenil generalizada.

Los hallazgos comunes para la periodontitis agresiva son:

- Pacientes clínicamente sanos, excepto por la presencia de la periodontitis.

- Rápida pérdida de inserción y destrucción ósea.

- Agregación familiar de los casos.

Los hallazgos secundarios que pueden presentarse o no son los siguientes:

- La cantidad de depósitos microbianos es inconsistente con la severidad de la destrucción periodontal de la enfermedad.

- Elevadas proporciones de A g $r$ e g a $t i b$ a $c t e r$ actinomytemcomitans $\mathrm{y}$ Porphyromonas gingivalis, en algunas poblaciones.

- Anormalidades en la fagocitosis.

- Respuesta elevada del fenotipo de los macrófagos, incluyendo niveles elevados de PGE2 e IL-1 $\beta$. 
- Etapas de progresión y detención de la pérdida de inserción y reabsorción ósea.(4)

La prevalencia de PA localizada encontrada en los países de Europa del este es 0,1 y $0,2 \%$; en Inglaterra 0,1\%; en Holanda 0,6\%; en Japón $0,47 \%$; en Arabia Saudita 0,42\%; en Nigeria entre $0,75 \%$ a $0,8 \%$; en varios lugares de EEUU con orígenes raciales mixtos entre $0,3 \%$ a $2,4 \%$; en Brasil 3,7\%, en Uganda es alta $26,8 \%$ en adolescentes de 12 a 16 años y 35,2\% en las edades de 17 a 19 años de edad (5). Susin y Albandar (6) encontraron en el 2005 que la prevalencia en el Brasil era 2,5\% en jóvenes de 14 a 19 años de edad, y que se incrementaba con la edad.

\section{Reporte de caso}

Paciente de sexo masculino de 13 años de edad, de raza mestiza, procedente de Lima, en Setiembre del 2009, acude a la Clínica Estomatológica de la Universidad Peruana Heredia, manifestando sangrado gingival, movilidad dentaria al momento del cepillado dental y separación de piezas dentarias anterosuperiores, lo que preocupa a la

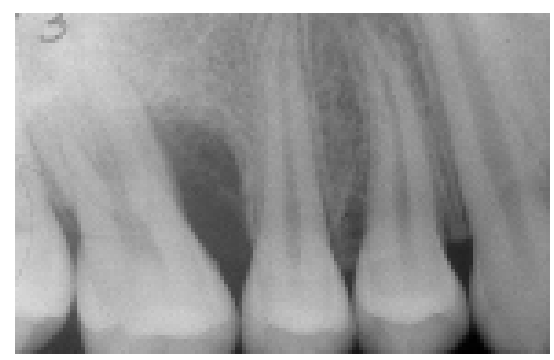

Fig. 1. Patrón de destrucción ósea con reabsorciones severas verticales a nivel de primeras molares superiores.
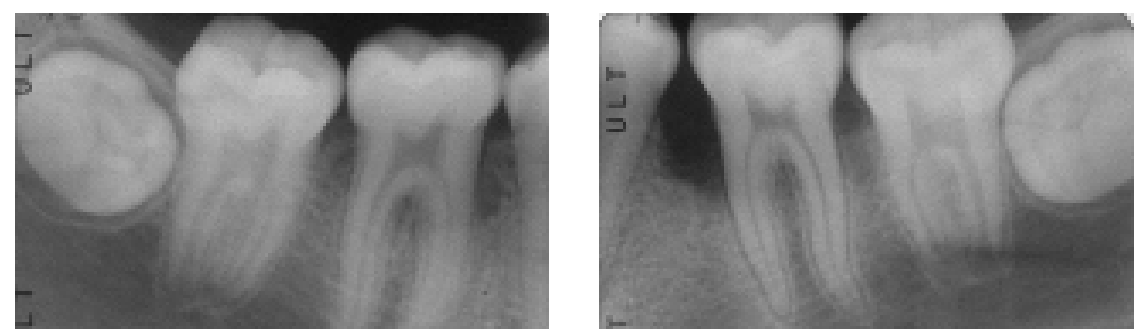

Fig. 2. Pérdida ósea horizontal a nivel de primeras molares inferiores. evaluada mediante el Índice gingival (Löe y Silness, 1963) con un valor promedio de 1,46 indicando que el paciente presentaba una inflamación gingival moderada.

En el periodontograma encontramos bolsas periodontales con profundidad de 4 a $9 \mathrm{~mm}$. Presencia de placa y/o cálculo en piezas 16, 15, 21, 25, 26, 27, 36, 31, 41. Movilidad dentaria (Indice de Miller, 1938) grado 1 , piezas $16,11,26$, y 41 . Sangrado al sondeado en piezas 12, 11, 21 , 22, 26, 36, 32, 31, 41, 42. Diastemas a nivel de las piezas $12 / 11,11 / 21$, $21 /$ 22, 31/41. Nivel de inserción clínica con pérdida entre 4 a $8 \mathrm{~mm}$.

Se realizó un estudio radiográfico seriado completo con radiografías periapicales y bite-wing de premolares y molares mediante la técnica del cono largo. Al examen radiográfico se observaron reabsorciones óseas severas verticales a nivel de piezas 16(M), 26(M), 36(M), 41(M) y horizontales a nivel de piezas 31(M), 41(M), 11(M), 21(M), 12(M), dando una imagen en espejo.

Al interpretar los hallazgos clínicos, radiográficos se observa en este adolescente sin compromiso sistémico, poca presencia de placa, pérdida de inserción clínica interproximal, reabsorciones óseas severas y horizontales localizadas especialmente a nivel de 1os primeros molares e incisivos, razón por la

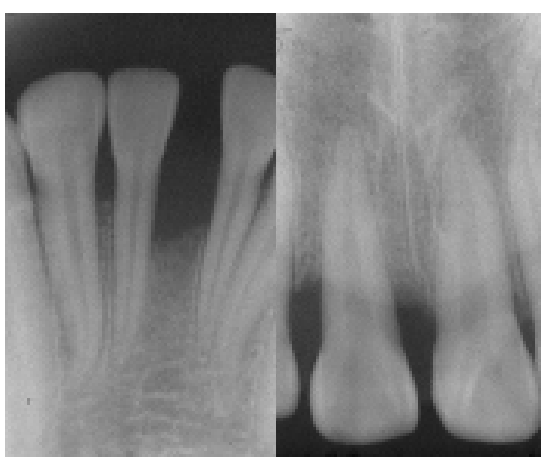

Fig. 3. Reabsorciones óseas horizontales a nivel de incisivos superiores e inferiores. 
cual se diagnóstica una periodontitis agresiva localizada (PAL). El paciente recibió instrucción de higiene oral para el control óptimo de placa bacteriana, profilaxis oral y terapia periodontal no quirúrgica mediante raspado y alisado radicular (RAR) para remover los depósitos microbianos, así como ajustes oclusales necesarios; acompañándose el tratamiento mecánico con medicación antibiótica adjunta; doxyciclina de $200 \mathrm{mg}$ inmediatamente post-RAR, mas $100 \mathrm{mg} 1 \mathrm{vez}$ al día por siete días; indicándose enjuagatorios de clorhexidina al 0,12\% después de cada comida.

Se realizaron controles semanales con el fin de mantener un estricto control de higiene oral. A las seis semanas post-RAR se realizó la reevaluación periodontal mediante la confección post-tratamiento de un nuevo periodontograma; encontrándose una reducción del nivel de inflamación gingival, disminución en la profundidad al sondeo de las bolsas periodontales, significativa ganancia de inserción clínica, buen control de placa bacteriana por parte del paciente.

\section{Discusión}

La periodontitis agresiva es una enfermedad rara, razón por la cual son muy pocos los casos que se re- portan. El riesgo de desarrollar periodontitis agresiva a tan temprana edad orienta a que estos pacientes sufrirán un mayor deterioro periodontal, es decir presentaran reabsorciones óseas severas, mayor pérdida del nivel de inserción clínica, bolsas periodontales más profundas, en un periodo de tiempo mas corto, el cual será más pronunciado en los sitios afectados inicialmente especialmente en aquellos pacientes que presentan PAL con un nivel socioeconómico bajo.

El significado de la periodontitis agresiva implica que los agentes etiológicos fueron capaces de producir niveles elevados de enfermedad detectados clínicamente en un periodo de corto de tiempo y, también implica una infección por una flora muy virulenta o un nivel alto de susceptibilidad por parte del paciente. (7)

Aggregatibacter actinomycetemcomitans (Aa), antes llamado Actinobacillus actinomycetemcomitans, es el microorganismo gramnegativo asociado a la PAL, por su incremento en su frecuencia de detección (90\%), produce diversos factores de virulencia los cuales evaden los mecanismos de defensa del huésped, asimismo tiene la capacidad de invadir las células epiteliales dentro de los
15 minutos post-infección $(8,9,10)$.

Los estudios demuestran que el raspado y alisado radicular (RAR) es una terapia efectiva usada con el fin de eliminar, controlar o disminuir los agentes microbianos presentes en la periodontitis, disminuyendo la carga bacteriana y consecuentemente la salud periodontal (11). Debido a los problemas técnicos para eliminar los microorganismos de la periodontitis y por su naturaleza infecciosa se recomienda el uso de agentes antibióticos y antimicrobianos adjunto al tratamiento de las periodontitis (12). La doxyciclina es uno de los antibióticos usados en el tratamiento de la periodontitis asociada al Aa, inhibiendo la síntesis proteíca, bacteriana mediante su unión a las subunidades ribosomales $30 \mathrm{~S}$ y $50 \mathrm{~S}$, cuyo objetivo principal es el de eliminar o alterar las colonias patógenas de estos microorganismos, asimismo reduce en el fluido crevicular gingival la actividad de las colagenasas (13), por ello es el antibiótico usado adjunto a la terapia mecánica que por sí sola no elimina al Aa.

El uso de un enjuagatorio a base de gluconato de clorhexidina al 0,12\% nos proporcionó un antiséptico con excelentes funciones bactericidas a fin de controlar la placa bacteriana e impedir su

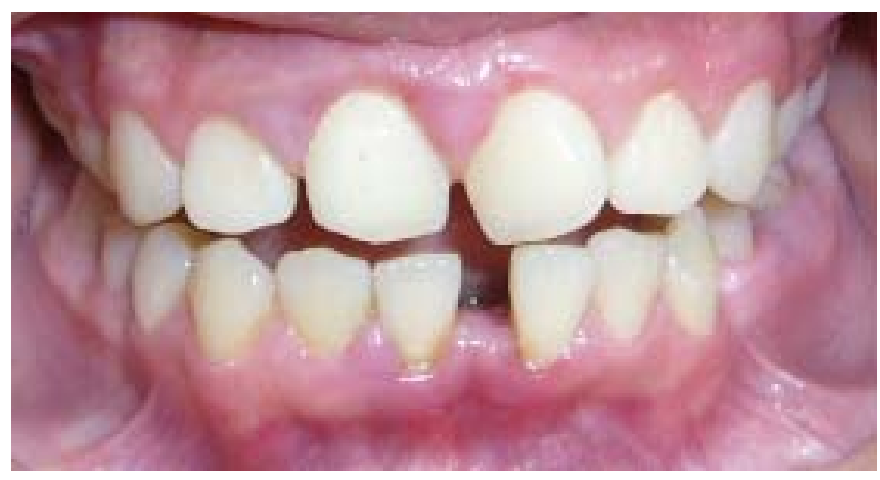

Fig. 4. Obsérvese el tejido gingival en la zona anterosuperior e inferior con coloración rojiza, textura lisa y brillante, edematosa, diastema muy marcado a nivel de incisivos superiores e inferiores y poca presencia de placa y/o cálculo dental.

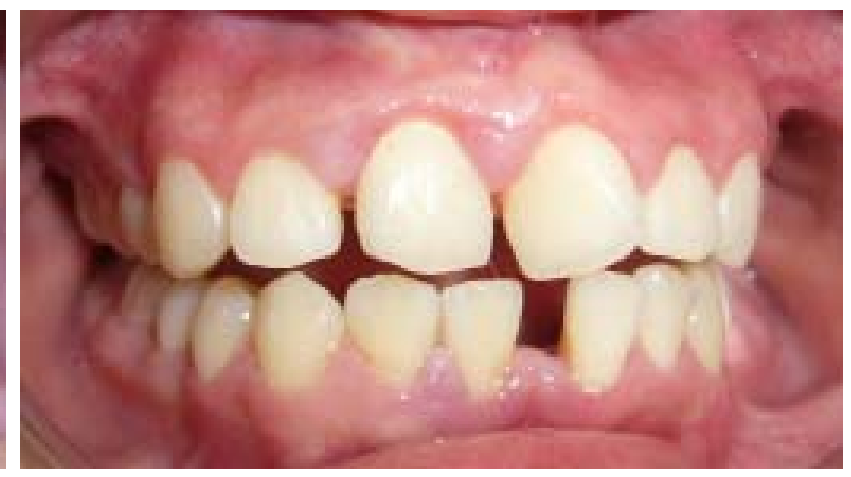

Fig. 5. Significativa reducción de los signos clínicos de inflamación gingival. 
reinfección (14).

En conclusión es de suma importancia detectar esta enfermedad periodontal de manera temprana ya que afecta a personas de grupos minoritarios, de escasos recursos económicos razón por la cual tienen una falta de acceso a los cuidados de salud tanto sistémicos como orales, lo que complica el control de la periodontitis agresiva.

\section{Referencias bibliográficas}

1. The American Academy of Periodontology (AAP). Consensus Report: Periodontal disease diagnostic ; 1989: 23- 4.

2. Armitage GC. Development of a classification system for periodontal diseases and conditions. Ann Periodontol. 1999; 4(1):1-6.

3. Parameter on aggressive periodontitis. American Academy of Periodontology. J Periodontol. 2000; 71(5 Suppl):867-9.

4. The American Academy of Periodontology (AAP). Consensus report: Aggressive periodontitis. Annals of Periodontol 1999; Dec 4 (1): 535.

5. Eres G, Saribay A, Akkaya M. Periodontal treatment needs and prevalence of localized aggressive periodontitis in a young Turkish population. J Periodontol. 2009; 80(6):940-4.

6. Susin C, Albandar JM. Aggressive periodontitis in an urban population in southern Brazil. J Periodontol. 2005; 76(3):468-75.

7. Tonetti MS, Mombelli A. Periodontitis agresiva. En: Kornman W, editor. Clinical Periodontics. Nueva York; 2001. p. 230- 52.

8. López M. Actinobacillus actinomycetemcomitans y Porphyromas ginngivalis en relación a las periodontitis agresivas. Rev Estomatol Herediana 2005; 15(2):178-83.

9. Carranza FA, Sznafder GH. Compendio de Periodoncia. 5 ed. Buenos Aires: Edit Médica Panamericana; 1996.

10. Yano-Higuchi K, Takamatsu N, He T, Umeda M, Ishikawa I. Prevalence of Bacteroides forsythus, Porphyromonas gingivalis and Actinobacillus actinomycetemcomitans in subgingival microflora of Japanese patients with adult and rapidly progressive periodontitis. J Clin Periodontol. 2000;
27(8):597-602.

11. Badersten A, Nilveus R, Egelberg J. Effect of nonsurgical periodontal therapy. II. Severely advanced periodontitis. J Clin Periodontol. 1984; 11(1):63-76.

12.Leiknes T, Leknes KN, Böe OE, Skavland RJ, Lie T. Topical use of a metronidazole gel in the treatment of sites with symptoms of recurring chronic inflammation. J Periodontol. 2007; 78(8):1538-44.

13. Lee JY, Lee YM, Shin SY, Seol YJ, Ku Y, Rhyu IC, Chung CP, Han SB. Effect of subantimicrobial dose doxycycline as an effective adjunct to scaling and root planing. J Periodontol. 2004; 75(11):1500-8.

14. Quirynen M, Mongardini C, de Soete M, Pauwels M, Coucke W, van Eldere J, van Steenberghe D. The rôle of chlorhexidine in the one-stage full-mouth disinfection treatment of patients with advanced adult periodontitis. Long-term clinical and microbiological observations. J Clin Periodontol. 2000; 27(8):578-89. 